\title{
COMPOSIÇÃO QUÍMICA E ATIVIDADES FOTOPROTETORA E ANTIRADICALAR IN VITRO DOS GALHOS DE
} Platonia insignis (CLUSIACEAE)

\author{
Andréia G. A. Silva ${ }^{a}$, Rodrigo de A. Moreira ${ }^{a}$, Renato Pinto de Sousa ${ }^{a}$, Evaldo dos S. M. Filho ${ }^{a}$, Marcia D. A. Veras ${ }^{a}$, \\ Mariana Helena Chaves ${ }^{a}$ e Sâmya D. L. Freitas ${ }^{\text {a*, (i) }}$ \\ aDepartamento de Química, Universidade Federal do Piauí, 64049-550 Teresina - PI, Brasil
}

Recebido em 14/03/2021; aceito em 07/04/2021; publicado na web em 06/05/2021

\begin{abstract}
CHEMICAL COMPOSITION AND PHOTOPROTECTIVE AND ANTIRADICAL ACTIVITIES OF THE BRANCHES OF Platonia insignis (CLUSIACEAE). The chemical study of the branches of Platonia insignis enabled the isolation of the biflavonoid morelloflavone and the identification, by mass spectrometry, of over 9 polyphenols, including eight biflavonoids, one benzophenone, and one xanthone. The in vitro biological tests of solar photoprotection and antiradical activities against the crude extract and Hex and AcOEt fractions showed relevant results, which confirm the high pharmaceutical potential of the species.
\end{abstract}

Keywords: Platonia insignis; biflavonoids; photoprotective activity; mass spectrometry.

\section{INTRODUÇÃO}

O uso de plantas com fins medicinais é provavelmente tão antigo quanto o aparecimento do próprio homem. A preocupação com a cura de doenças sempre se fez presente ao longo da história da humanidade. ${ }^{1}$ No Brasil, o conhecimento das propriedades de plantas medicinais é uma das maiores riquezas da cultura indígena, uma sabedoria tradicional que é passada de geração em geração. ${ }^{2}$ As plantas medicinais sempre foram objeto de estudo, buscando-se novas fontes para obtenção de princípios ativos (metabolitos secundários), responsáveis por sua ação farmacológica ou terapêutica. ${ }^{3}$

A família Clusiaceae Lindl., também conhecida como Guttiferae, apresenta uma distribuição pantropical, incluindo cerca de 55 gêneros e 1.200 espécies. ${ }^{4,5}$ No Brasil, encontra-se representada por 12 gêneros ( 1 endêmico); 126 espécies (42 endêmicas), além de 4 subespécies que podem ser encontradas como arbustos, árvores ou lianas. ${ }^{6}$ A família não é endêmica do país, no entanto, é nativa de grande parte dele, com ocorrências confirmadas nas regiões Norte, Nordeste, CentroOeste, Sudeste e Sul. ${ }^{6,7}$

Estudos realizados com plantas da família Clusiaceae identificaram constituintes químicos pertencentes a classes e subclasses de metabolitos como cumarinas, xantonas, benzofenonas, biflavonoides e triterpenos. ${ }^{8-11}$ Os biflavonoides são umas das sub-classes mais abundantes, considerados como marcadores taxonômicos da família. ${ }^{9}$

O gênero Platonia Mart. tem como sinônimo heterotípico Aristoclesia Coville e apresenta uma única espécie (Platonia insignis). É um gênero nativo, não endêmico do Brasil, com distribuição nas regiões Norte (Amazônia, Pará, Roraima) e Nordeste (Maranhão e Piauí), além de outros países como Peru, Venezuela, Colômbia e Guiana Francesa. ${ }^{6,12}$

A espécie Platonia insignis é conhecida popularmente como bacurizeiro ou bacuri. É uma planta madeireira, de frutos comestíveis, bastante saboreada pela população, que utiliza a polpa do fruto para fabricação de sucos, sorvetes, doces e outros. ${ }^{5} \mathrm{~A}$ espécie é pertencente à família Clusiaceae e nativa do cerrado brasileiro, também apresenta sinonímias como Aristoclesia esculenta Stuntz, Moronobea esculenta Arruda e Platonia esculenta Oken. ${ }^{6}$ O óleo das sementes é empregado na medicina popular no tratamento de problemas de pele, tais como queimaduras, feridas, e no tratamento de dores lombares, inflamações e cicatrização. ${ }^{13}$

Atualmente existe na literatura registros de estudos fitoquímicos das cascas das sementes, dos frutos e do caule, da polpa, da semente e das flores de P. insignis Mart. ${ }^{11,14-16}$ Estudos farmacológicos também foram realizados com a espécie, como atividade antioxidante in vitro, citotóxica, anticolinesterásica, antimicrobiana, anticonvulsivante, cicatrizante, antileishmania e gastroprotetora. ${ }^{5,8,11,13,14,17-19}$

A revisão bibliográfica de $P$. insignis mostra que a espécie é promissora para a indústria farmacêutica e biotecnológica, estimulando estudos mais aprofundados. Apesar da existência de estudos químicos e farmacológicos com as sementes e cascas dos frutos de $P$. insignis, não foram encontrados trabalhos relatados na literatura com os galhos. Assim, considerando o potencial verificado a partir dos resultados anteriores e devido à grande quantidade de compostos fenólicos e com potencial de atividade antioxidante in vitro frente ao radical DPPH, o presente trabalho teve como objetivo realizar uma investigação química e a avaliação do potencial fotoprotetor, devido à alta incidência de radiação solar no Cerrado e antioxidante dos galhos de Platonia insignis Mart. (Clusiaceae), contribuindo ainda mais para o conhecimento científico da espécie.

\section{PARTE EXPERIMENTAL}

\section{Instrumentação e procedimentos gerais}

Os solventes etanol e metanol utilizados na extração e fracionamento foram de grau analítico (Synth). $\mathrm{Na}$ análise por espectrometria de massa foram de grau HPLC e a água ultrapura (resistividade 18.2 $\mathrm{M} \Omega$ ) foi obtida em um sistema de purificação. Os reagentes utilizados na análise de teor de fenóis totais, atividade antioxidante e fotoprotetora foram Folin-Ciocalteu (Merck), carbonato de sódio (Synth), radical 2,2-difenil-1-picril-hidrazila $(\mathrm{DPPH} \bullet)($ Sigma), rutina (Sigma), BHT (Vetec).

As medidas de absorção foram feitas usando espectrofotômetro UV-Vis Lambda 25 (PerkinElmer, Waltham, MA, USA). Os espectros de massas foram obtidos por inserção direta em Espectrômetro de Massas com analisador Ion trap (ITMS, AMAZONAS X, Bruker Daltonics) equipado com fonte de ionização por eletrospray (ESI). 


\section{Material vegetal}

Os galhos da espécie Platonia insignis foram coletados em 17 de janeiro de 2016, na cidade de Timon no Maranhão, nas coordenadas $\mathrm{S}$ 04 50 ' 56,6" e W 042 05 ' 04,8". A exsicata da espécie com número TEPB 31.718 e cadastro SisGen ACB65D0 encontra-se depositada no Herbário Graziela Barroso - UFPI, na cidade de Teresina.

\section{Obtenção do extrato etanólico, frações e sufrações}

Os galhos (906 g) foram limpos por meio de água corrente, secos à temperatura ambiente e triturados por moinho de facas. $\mathrm{O}$ material vegetal foi submetido à maceração com etanol por 6 vezes durante oito dias. O solvente foi removido em evaporador rotatório sob pressão reduzida e liofilizado para a obtenção do extrato bruto $(57,88 \mathrm{~g})$.

Uma alíquota $(25,30 \mathrm{~g})$ do extrato bruto $(\mathrm{EEtOH})$ dos galhos de $P$. insignis foi submetida à extração líquido-líquido por meio de suspensão em MeOH/H $\mathrm{H}_{2} \mathrm{O}(1: 2)(100 \mathrm{~mL})$ e pelos solventes orgânicos hexano e acetato de etila, obtendo-se respectivamente frações: hexano (Hex) (10,04 g), acetato de etila (AcOEt) $(12,04 \mathrm{~g})$ e fração residual, hidroalcoólica (HA) $(1,45 \mathrm{~g})$.

A fração $\mathrm{AcOEt}(10 \mathrm{~g})$ foi adsorvida em uma coluna cromatográfica de gel sílica, eluída com clorofórmio/metanol em ordem crescente de polaridade. Foram coletadas 71 frações e reunidas em 33 subfrações após análise por CCDC, na qual se destacou a FAc21 (210 mg) apresentando um precipitado branco, com uma única mancha amarela intensa em CCD, característico de substância pura.

\section{Análise por ESI-IT/MS ${ }^{\mathrm{n}}$ do extrato bruto, fração AcOEt e sufração FAc21 dos galhos de $P$. insignis}

As análises realizadas por ESI-IT/MS ${ }^{\mathrm{n}}$ do extrato bruto, fração AcOEt e subfração FAc21 dos galhos de $P$. insignis, foram obtidos através da inserção direta (ID) via bomba de seringa, em espectrômetro de massas com analisador armadilha de íons (ITMS, AmaZon X, Bruker Daltonics), em fluxo contínuo de $5 \mu \mathrm{L} \mathrm{min}{ }^{-1}$, nas faixas de massa $m / z 100$ a 1500 , em metanol/água (1:1). As amostras foram ionizadas por electrospray (ESI) e as fragmentações foram obtidas em múltiplos estágios $\left(\mathrm{MS}^{\mathrm{n}}\right)$, em uma interface do tipo Ion trap (IT). O modo negativo foi escolhido para a geração e análise de todos os espectros, a voltagem do capilar $4,5 \mathrm{kV}$, nebulizador de nitrogênio com fluxo de $5 \mathrm{~L} \mathrm{~min}^{-1}$ e temperatura da fonte de $220^{\circ} \mathrm{C}$ a uma pressão de 10 psi para o nebulizador de nitrogênio. Antes de cada análise o ESI-IT-MS foi calibrado com formiato de sódio, sendo o erro inferior a $1 \mathrm{ppm}$. $\mathrm{O}$ tratamento dos espectros de massas foi realizado no software Compass 1.3 Data Analysis (versão 4.0, Bruker Daltonics).

\section{Espectroscopia de Ressonância Magnética Nuclear de ${ }^{1} \mathrm{H}$}

A subfração FAc21 foi analisada por espectroscopia de RMN de hidrogênio $\left({ }^{1} \mathrm{H}\right)$ solubilizada em metanol deuterado. $\mathrm{O}$ espectro foi obtido em espectrômetro Varian INOVA - modelo 400, operando a $400 \mathrm{MHz}\left({ }^{1} \mathrm{H}\right)(9,4$ Tesla).

\section{Determinação do teor de fenóis totais}

O teor de fenóis totais foi determinado seguindo a metodologia descrita por Uriu Martins et al. ${ }^{20} \mathrm{O}$ extrato bruto e frações Hex e AcOEt foram solubilizadas em água destilada com auxílio de 5\% de DMSO em concentrações de $250 \mu \mathrm{g} \mathrm{mL}^{-1}$. Dentre as soluções preparadas, foram retiradas alíquotas de $0,5 \mathrm{~mL}$ e transferidas para tubos de ensaio, adicionados $8 \mathrm{~mL}$ de água destilada e $0,5 \mathrm{~mL}$ do reagente Folin Ciocalteau 20\% (v:v). Em seguida, a solução foi homogeneizada em agitador tipo vortex, após 3 minutos, acrescido 1 $\mathrm{mL}$ de solução de carbonato de sódio $\left(\mathrm{Na}_{2} \mathrm{CO}_{3}\right)$ a $20 \%$ (m:v) e após 1 $\mathrm{h}$ em banho maria a $37^{\circ} \mathrm{C}$, foram realizadas as leituras das densidades óticas em espectrofotômetro à $720 \mathrm{~nm}$. Utilizou-se como padrão de referência o ácido gálico da marca Sigma ${ }^{\circledR}$ para construção da curva de calibração que foi expressa por $A=0,0057 \mathrm{C}+0,014$, com coeficiente de correlação linear igual a 0,999 , em que "C" é a concentração do ácido gálico e " $A$ " é absorbância. A partir da equação da reta obtida na curva de calibração, realizou-se o cálculo do teor de fenóis totais, expresso em EAG (equivalente ao ácido gálico $\mathrm{mg}^{-1}$ ) de amostra. Todas as análises foram realizadas em triplicata. Para construção dos gráficos e análises estatísticas, utilizou-se o programa Origin $8.0^{\circledR}{ }^{21}$

Determinação do fator de proteção solar (FPS) e comprimento de onda máximo e absorbância do extrato, frações e subfração FAc21

A determinação do Fator de Proteção Solar (FPS) foi baseado no método espectrofotométrico de amostras em solução adaptado por Mansur. ${ }^{22}$ Soluções contendo o extrato bruto, frações e subfração FAc21, foram preparadas com as respectivas concentrações 5, 25, 50 e $100 \mathrm{mg} \mathrm{mL}^{-1}$, em etanol. As mesmas, foram submetidas à leitura de suas absorbâncias utilizando cubetas de quartzo em um espectrofotômetro (UV-Vis PerkinElmer - Lambda 25) nas faixas de 200 a $400 \mathrm{~nm}$, com intervalos de leituras de $5 \mathrm{~nm}$. Em seguida, foram substituídos os valores de absorbância obtidos, na equação adaptada por Mansur e colaboradores (1986) (Equação 1), que relaciona produto entre o efeito eritematógeno (EE) e a intensidade da radiação (I), que é constante em cada comprimento de onda (Tabela 1). ${ }^{22}$ Os testes foram realizados em triplicatas..$^{23}$

$$
\mathrm{FPS}=\mathrm{FC} \times \sum_{290}^{320} \mathrm{EE}(\lambda) \times \mathrm{I}(\lambda) \times \operatorname{Abs}(\lambda)
$$

em que: $\mathrm{FC}=$ fator de correção igual a $10 ; \operatorname{EE}(\lambda)=$ efeito eritematógeno da radiação solar em cada comprimento de onda $(\lambda)$; $\mathrm{I}(\lambda)=$ intensidade da radiação solar em cada comprimento de onda $(\lambda) \operatorname{Abs}(\lambda)=$ leitura da absorbância obtida da amostra em cada comprimento de onda $(\lambda)$.

Tabela 1. Ponderação empregada no cálculo do fator de proteção solar por espectrofotometria. ${ }^{24}$

\begin{tabular}{cc}
\hline Comprimento de onda $(\mathrm{nm})$ & $\begin{array}{c}\text { EE x I (normalizado) } \\
\text { Valores relativos }\end{array}$ \\
\hline 290 & 0,0150 \\
\hline 295 & 0,0817 \\
300 & 0,2874 \\
305 & 0,3278 \\
310 & 0,1864 \\
315 & 0,0839 \\
320 & 0,0180 \\
\hline Total & 1,0002 \\
\hline
\end{tabular}

\section{Atividade antioxidante frente ao radical DPPH}

O ensaio da atividade antioxidante foi realizado frente ao radical DPPH (2,2-difenil-1-picril-hidrazil) de acordo com a metodologia descrita pela literatura, com algumas adaptações. ${ }^{25}$ Para o extrato bruto, frações e subfração FAc21 dos galhos de $P$. insignis foram utilizados $12,5 \mathrm{mg}$ de cada em triplicata, e preparado soluções com metanol nas seguintes concentrações: $250 \mu \mathrm{g} \mathrm{mL}^{-1}$ que foram diluídas 
nas concentrações de 200, 150, 100, 50 e $25 \mu \mathrm{g} \mathrm{mL}{ }^{1}$. Posteriormente, foi preparado uma solução estoque do radical DPPH na concentração de $40 \mu \mathrm{g} \mathrm{mL}^{-1}$ que foi protegida da luz.

A leitura das absorbâncias $(A)$ das misturas reacionais $(0,3 \mathrm{~mL}$ da solução da amostra e $2,7 \mathrm{~mL}$ da solução estoque de DPPH, na concentração de $40 \mu \mathrm{g} \mathrm{mL}^{-1}$ ), foram feitas a $516 \mathrm{~nm}$, em triplicata, no $1^{\circ}, 5^{\circ}$ e $10^{\circ}$ minuto de análise, a cada 10 min até completar 30 min. Utilizando-se como branco a solução metanólica da amostra $(0,3 \mathrm{~mL})$ e metanol (2,7 mL). A equação da curva analítica, foi construída com o DPPH nas concentrações de 35, 30, 25, 20, 15, 10, 5 e $1 \mu \mathrm{g} \mathrm{mL}^{-1}$, onde a $A=33,227 c+1,0607$, com coeficiente de correlação linear $\mathrm{r}=$ 0,9997. A partir da equação da curva do radical DPPH e dos valores de absorbância no tempo de 30 min para cada concentração testada, foram determinados os percentuais de DPPH remanescentes $\left(\% \mathrm{DPPH}_{\mathrm{REM}}\right)$. $\mathrm{O}$ percentual de atividade antioxidante (\%AA) do extrato, frações e subfração FAc21 foram determinados a partir dos valores de suas absorbâncias medidas nas concentrações de 25, 50, 100, 150, 200 e 250 $\mu \mathrm{g} \mathrm{mL} \mathrm{mL}^{-1}$ no tempo de 30 minutos e calculado de acordo com a equação, $\% A A=100-\left([D P P H]_{\text {Rem }} /[D P P H]_{t 0}\right) \times 100$. Os resultados foram expressos pelo valor de $\mathrm{CE}_{50}$, concentração eficiente em $\mu \mathrm{g} \mathrm{mL}{ }^{-1} \mathrm{da}$ amostra capaz de reduzir em $50 \%$ a concentração do radical livre DPPH.

\section{RESULTADOS E DISCUSSÃO}

\section{Análise por ESI-IT/MS ${ }^{\mathrm{n}}$ do extrato bruto, fração AcOEt e subfração FAc21 dos galhos de Platonia insignis}

Os espectros de íons totais obtidos da análise por ESI-IT/MS ${ }^{\mathrm{n}}$ do extrato, fração AcOEt e subfração FAc21 apresentaram uma grande variedade de íons, que ao serem detectados, sugeriu a presença de diversos metabolitos como: biflavonoides, benzofenona e xantona na composição química. A Figura 1 apresenta os espectros de massas obtidos em modo negativo [ESI (-)] do extrato bruto (A), fração AcOEt (B) e subfração FAc21 (C), apresentando sinais de maior intensidade os íons com $\mathrm{m} / \mathrm{z}, 601, \mathrm{~m} / \mathrm{z}, 555, \mathrm{~m} / \mathrm{z}, 557$ e $\mathrm{m} / \mathrm{z} 573$.

A identificação dos compostos da espécie foi obtida com base no comportamento espectral de massa das fragmentações em experimentos de $\mathrm{MS}^{\mathrm{n}}$, pela seleção e fragmentação do íon precursor e comparações com a literatura. Portanto, um total de 11 picos de maior intensidade foram detectados, os mesmos podem ser observados na Tabela 2. Os compostos $\mathbf{1}$ - $\mathbf{1 1}$ estão presentes no extrato bruto e na fração AcOEt, já a subfração FAc21 apresentou somente o composto $\mathbf{2}$, confirmando seu isolamento (Figura 2).
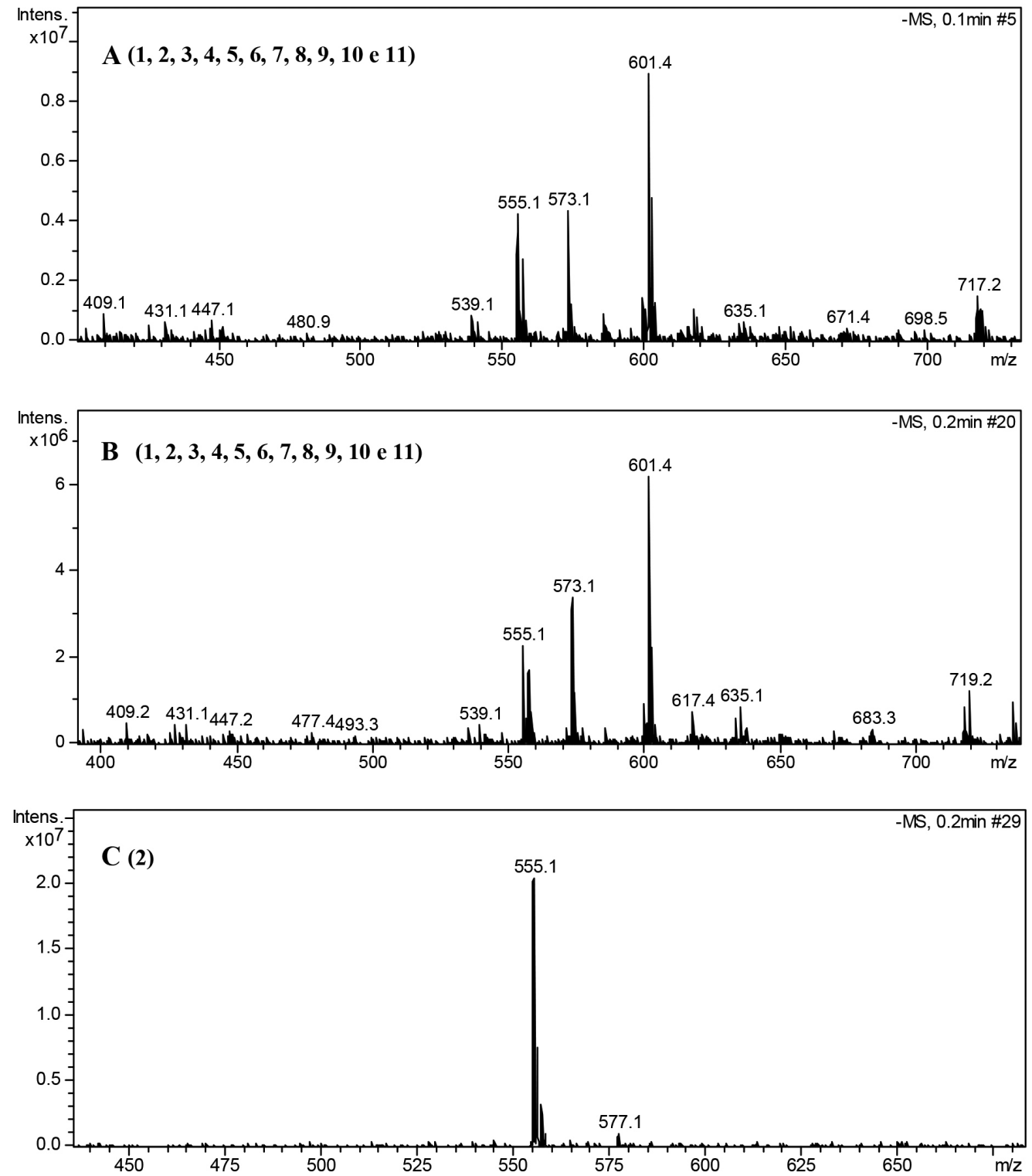

Figura 1. Espectro de massas [ESI (-)] do extrato (A), fração AcOEt (B) e subfração FAc21 (C) dos galhos de P. insignis 
Tabela 2. Identificação dos constituintes químicos do extrato, fração AcOEt e substância isolada dos galhos de $P$. insignis

\begin{tabular}{|c|c|c|c|c|c|c|}
\hline $\mathrm{N}^{\circ}$ & Constituinte química & $\begin{array}{l}\text { Fórmula } \\
\text { molecular }\end{array}$ & $\begin{array}{c}\text { Massa } \\
\text { molecular }\end{array}$ & $\begin{array}{r}{[\mathrm{M}-\mathrm{H}]^{-}} \\
(m / z)\end{array}$ & $\mathrm{ESI} \mathrm{MS}^{\mathrm{n}}(\%$ pico base$)$ & Referências \\
\hline 1 & $\alpha$-Mangostin & $\mathrm{C}_{24} \mathrm{H}_{26} \mathrm{O}_{6}$ & 410 & 409 & $\begin{array}{l}\mathrm{MS}^{2}[409]: 351(100), 394(17,26), 339(21,18), 377(2,98) \\
\operatorname{MS}^{3}[409 \rightarrow 351]: 295.9(100), 335.9(75,61), 307(28,28),\end{array}$ & 29 \\
\hline 2 & Morelloflavona & $\mathrm{C}_{30} \mathrm{H}_{20} \mathrm{O}_{11}$ & 556 & 555 & $\begin{array}{c}\mathrm{MS}^{2}[555]: 429(100), 403(4,16) \\
\mathrm{MS}^{3}[555 \rightarrow 429]: 401(100), 356.9(17,61), 294.5(11,25)\end{array}$ & 26 \\
\hline 3 & GB-2a & $\mathrm{C}_{30} \mathrm{H}_{22} \mathrm{O}_{11}$ & 558 & 557 & $\begin{array}{c}\operatorname{MS}^{2}[557]: 431(100), 403(6,0) \\
\operatorname{MS}^{3}[557 \rightarrow 431]: 294.9(100), 329.9(14,29), 403(17,35), 413(24,21)\end{array}$ & 26 \\
\hline 4 & GB-2 & $\mathrm{C}_{30} \mathrm{H}_{22} \mathrm{O}_{12}$ & 574 & 573 & $\begin{array}{c}\mathrm{MS}^{2}[573]: 447(100), 419(13,78) \\
\mathrm{MS}^{3}[573 \rightarrow 447]: 419(100), 294.9(10,76)\end{array}$ & 26 \\
\hline 5 & Garcinilipitona FC & $\mathrm{C}_{38} \mathrm{H}_{50} \mathrm{O}_{6}$ & 602 & 601 & $\begin{array}{l}\mathrm{MS}^{2}[601]: 465.3(100), 409(66,57), 557(27,60) \\
\quad \mathrm{MS}^{3}[601 \rightarrow 273]: 273(100), 397.3(25,73)\end{array}$ & 14 \\
\hline 6 & $\begin{array}{l}\text { Morelloflavona- } 7^{\prime \prime}-O \text { - } \\
\text { sulfato }\end{array}$ & $\mathrm{C}_{30} \mathrm{H}_{20} \mathrm{O}_{14} \mathrm{~S}$ & 636 & 635 & $\begin{array}{c}\operatorname{MS}^{2}[635]: 429(100), 555(81,87), 499(16,46) \\
\operatorname{MS}^{3}[635 \rightarrow 429]: 401(100), 295(16,65), 357(19,0)\end{array}$ & 26 \\
\hline 7 & GB-2a-sulfato & $\mathrm{C}_{30} \mathrm{H}_{18} \mathrm{O}_{14} \mathrm{~S}$ & 638 & 637 & $\begin{array}{c}\operatorname{MS}^{2}[637]: 431(100), 557(30,11) \\
\operatorname{MS}^{3}[637 \rightarrow 431]: 294.9(100), 413(17,11)\end{array}$ & 26 \\
\hline 8 & Fukugisídio & $\mathrm{C}_{36} \mathrm{H}_{30} \mathrm{O}_{16}$ & 718 & 717 & $\begin{array}{l}\mathrm{MS}^{2}[717]: 565(100), 429(57,51), 591(34,18) \\
\quad \mathrm{MS}^{3}[717 \rightarrow 565]: 403(100), 445(9,24)\end{array}$ & 26 \\
\hline 9 & $\begin{array}{l}\text { GB-2a- } O \text { - } \\
\text { glicopiranosídio }\end{array}$ & $\mathrm{C}_{36} \mathrm{H}_{32} \mathrm{O}_{16}$ & 720 & 719 & $\begin{array}{l}\mathrm{MS}^{2}[719]: 431(100), 557(24,90), 593(8,16) \\
\quad \operatorname{MS}^{3}[719 \rightarrow 431]: 294.3(100), 413(16,15)\end{array}$ & 26 \\
\hline 10 & $\begin{array}{l}\text { GB2-7"'-O- } \\
\text { glicopiranosídeo }\end{array}$ & $\mathrm{C}_{36} \mathrm{H}_{32} \mathrm{O}_{17}$ & 736 & 735 & $\begin{array}{c}\mathrm{MS}^{2}[735]: 447(100), 573(11,84) \\
\mathrm{MS}^{3}[735 \rightarrow 447]: 429(100), 419(27,60), 268.9(14,83)\end{array}$ & 26 \\
\hline 11 & NI & - & 762 & 761 & $\mathrm{MS}^{2}[761]: 679(100), 615.3(87,25), 597.2(73,28)$ & - \\
\hline
\end{tabular}

NI: composto não identificado.<smiles>COc1cc(O)c(OC)c2c(O)cc(CC=C(C)C)c(O)c1c2=O</smiles><smiles>C=C(C)CCC1C[C@@H]2CC3(CC=C(C)C)C(=O)C(CC=C(C)C)=C(O)C(C(=O)c4ccc(O)c(O)c4)(C3=O)C2(C)C(=O)C1C(C)C</smiles><smiles>[R3]c1ccc(-c2cc(=O)c3c(O)cc([R])c(C4C(=O)c5c(O)cc(O)cc5OC4c4ccc(O)c([R])c4)c3o2)cc1[R]</smiles>

2: $\mathrm{R}_{1}=\mathrm{R}_{2}=\mathrm{H}, \mathrm{R}_{3}=\mathrm{R}_{4}=\mathrm{OH}$

6: $\mathrm{R}_{1}=\mathrm{H}, \mathrm{R}_{2}=\mathrm{SO}_{3} \mathrm{H}, \mathrm{R}_{3}=\mathrm{R}_{4}=\mathrm{OH}$

8: $\mathrm{R}_{1}=\mathrm{H}, \mathrm{R}_{3}=$ OGlc, $\mathrm{R}_{2}=\mathrm{H}$, $\mathrm{R}_{4}=\mathrm{OH}$

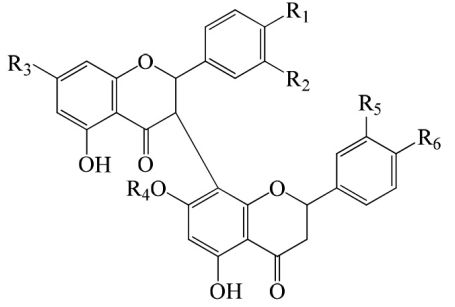

3: $\mathrm{R}_{4}=\mathrm{H}, \mathrm{R}_{1}=\mathrm{R}_{2}=\mathrm{R}_{3}=\mathrm{R}_{5}=\mathrm{R}_{6}=\mathrm{OH}$

7: $\mathrm{R}_{1}=\mathrm{OH}, \mathrm{R}_{2}=\mathrm{H}, \mathrm{R}_{3}=\mathrm{R}_{5}=\mathrm{R}_{6}=\mathrm{OH}$,

$\mathrm{R}_{4}=\mathrm{SO}_{3} \mathrm{H}$

9: $\mathrm{R}_{1}=\mathrm{R}_{3}=\mathrm{R}_{5}=\mathrm{R}_{6}=\mathrm{OH}, \mathrm{R}_{2}=\mathrm{H}$,

$\mathrm{R}_{4}=\mathrm{Glc}$

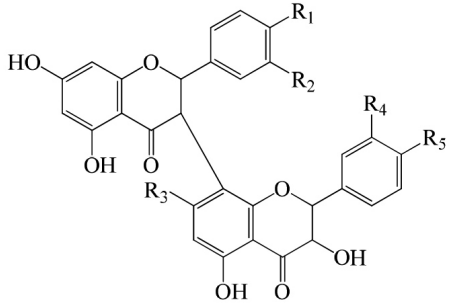

4: $\mathrm{R}_{1}=\mathrm{R}_{3}=\mathrm{R}_{4}=\mathrm{R}_{5}=\mathrm{OH}, \mathrm{R}_{2}=\mathrm{H}$

10: $R_{1}=R_{4}=R_{5}=O H, R_{2}=H$,

$\mathrm{R}_{3}=$ OGlc

Figura 2. Constituintes químicos identificados no extrato, fração AcOEt e subfração FAc21 dos galhos de P. insignis

\section{Biflavonoides}

Os biflavonoides dos galhos de $P$. insignis foram os constituintes majoritários identificados, totalizando 8 compostos. Na família Clusiaceae é relatado a presença de uma variedade desses metabolitos, no qual, são agrupados em quatros tipos principais: GB1, GB-1a, morelloflavona e amentoflavona. ${ }^{9}$ Neste trabalho, foram identificados na espécie três tipos de biflavonoides de Clusiaceae (Figura 2).

\section{Tipo Morelloflavona}

Os compostos 2, 6 e 8 foram identificados como biflavonoides do tipo morelloflavona devido ao padrão de ligação de seus monômeros (flavanona-(3 $\left.\rightarrow 8^{\prime \prime}\right)$-flavona). ${ }^{9,10} \mathrm{O}$ composto 2 , presente no EEtOH, fração AcOEt e como único pico na subfração FAc21 apresentou íon molecular em $\mathrm{m} / z 555$ [M-H] sendo identificado como morelloflavona (naringenina- $\left(3 \rightarrow 8^{\prime \prime}\right)$ - luteolina) ou Fukugentina, e confirmado pelo produto da via de fragmentação $\mathrm{MS}^{2} \mathrm{em} m / z, 429$ [M-H-126], 
correspondendo a perda $-\mathrm{C}_{6} \mathrm{H}_{6} \mathrm{O}_{3}$ do floroglucinol (-126 Da), devido à clivagem do anel $\mathrm{C}$ no monômero de naringerina. Na fragmentação em $\mathrm{MS}^{3}$, o composto apresentou um íon em $\mathrm{m} / z, 401$ [M-H-28] pela perda neutra de $\mathrm{CO}$ no anel $\mathrm{C}$ do monômero de naringerina (Figura $2 \mathrm{~S}$ ). Este constituinte é considerado um marcador quimiossistemático de Clusiaceae (Figura 3a). ${ }^{9}$
O íon molecular em $m / z, 635$ [M-H]', pertencente ao composto 6 , foi identificado como morelloflavona-7"'- $O$-sulfato. ${ }^{9,26}$ Esse composto apresenta íon fragmento $\mathrm{MS}^{2}$ em $\mathrm{m} / \mathrm{z} 429$ [M-H-206] pela perda de $-\mathrm{C}_{6} \mathrm{H}_{6} \mathrm{O}_{3}$ e $-\mathrm{SO}_{3}$ e o íon em $\mathrm{m} / z, 555$ (característico do composto morelloflavona) e $\mathrm{MS}^{3} \mathrm{em} \mathrm{m} / \mathrm{z}, 401$ [M-H-28] (Figura 6S) pela perda de uma molécula de $\mathrm{CO} .{ }^{26} \mathrm{O}$ composto 8 apresentou o íon molecular
MS

a)<smiles>CCCCC1(c2ccc(O)cc2)Oc2cc(O)cc(O)c2C1c1c(O)cc(O)c2c(=O)cc(-c3ccc(O)cc3)oc12</smiles>

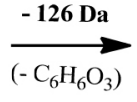

$\mathrm{MS}^{2}$

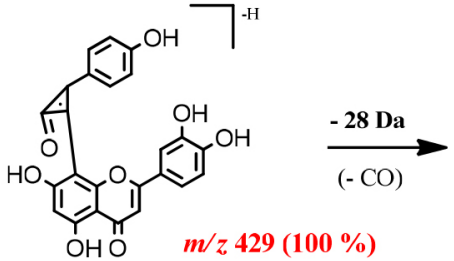

$m / z 555$
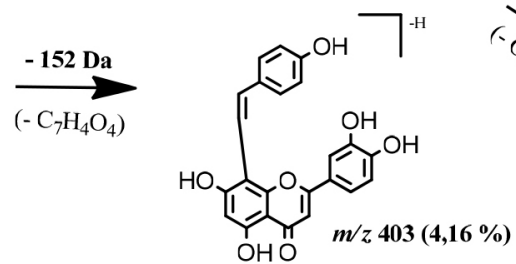

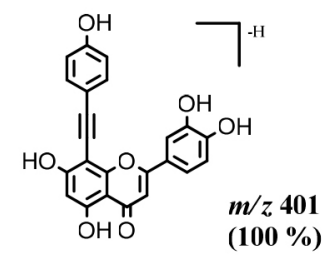

$\mathbf{M S}^{3}$

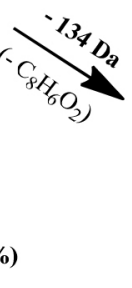

b)

MS

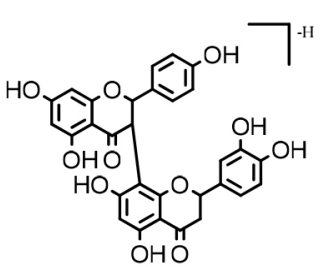<smiles>Oc1ccccc1</smiles>

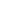

MS
MS $^{3}$

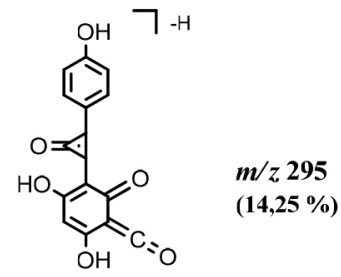

c)
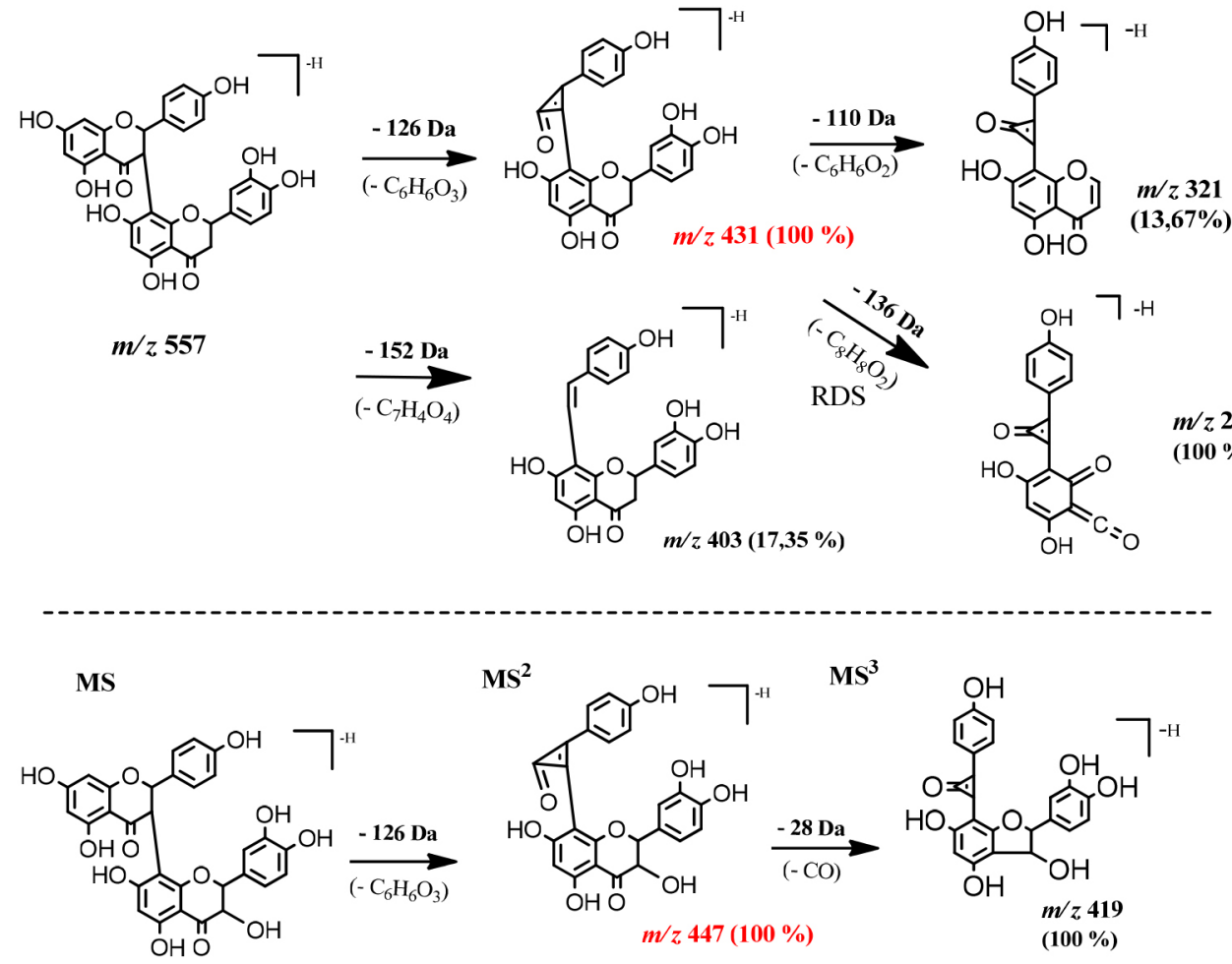

$m / z 557$
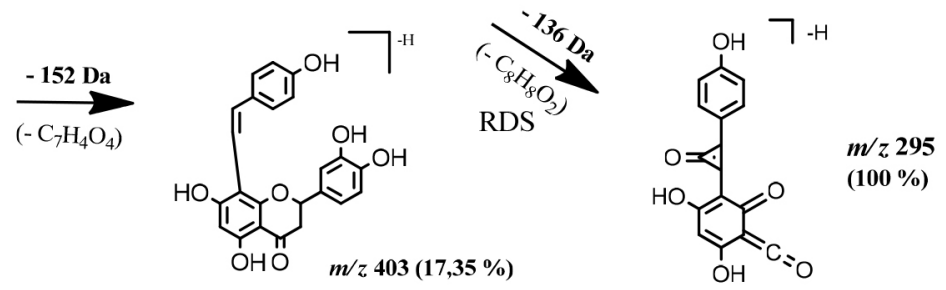
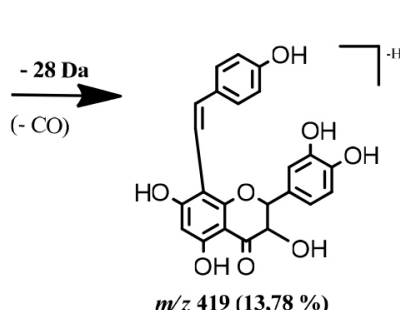

$\mathbf{M S}^{3}$
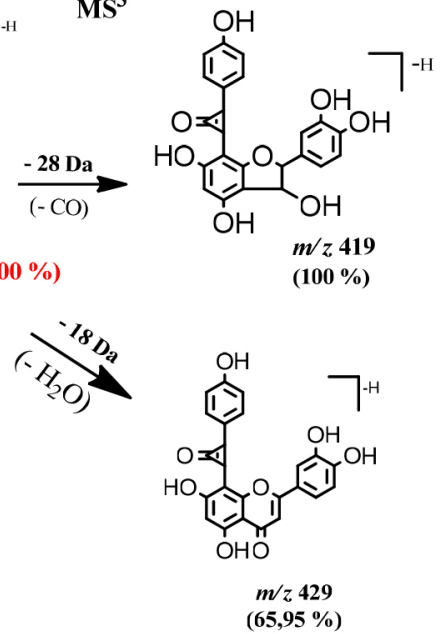

Figura 3. Proposta de vias de fragmentação $M S^{n}$ para os biflavonoides do tipo morelloflavona (a), GB-1a (b) e GB1 (c) de Platonia insignis 
em $m / z 717$ [M-H] e foi identificado como fukugisídeo, um derivado glicosilado da morelloflavona, apresentando como íon fragmento $\mathrm{MS}^{2}$ $\mathrm{em} m / z 565$ [M-H-152] com perda de $-\mathrm{C}_{7} \mathrm{H}_{4} \mathrm{O}_{4}$ referente à clivagem do anel $\mathrm{A}$ e parte do anel $\mathrm{C}$ do monômero de naringerina, e íon produto $\mathrm{MS}^{3} \mathrm{em} \mathrm{m} / z, 403$ [M-H-162] com perda de glicose (-162 Da) no anel A do Flavonoide II do monômero de luteolina (Figura 8S). ${ }^{26}$

\section{Tipo GB-1a}

Os compostos 3, 7 e 9 foram identificados como biflavonoides do tipo GB-1a (biflavonoide do gênero Garcinia), que apresentam padrões de ligação dos seus monômeros (flavanona ( $\left.3 \rightarrow 8^{\prime \prime}\right)$-flavanona). ${ }^{9} \mathrm{O}$ composto 3 apresentou pico do íon molecular desprotonado em $\mathrm{m} / \mathrm{z}$ $557[\mathrm{M}-\mathrm{H}]^{-}$e foi identificado como GB-2a (naringenina- $\left(3 \rightarrow 8^{\prime \prime}\right)$ eriodictiol), e o seu íon produto $\mathrm{MS}^{2} \mathrm{em} \mathrm{m} / z 431$ [M-H-126] ${ }^{-}$com a perda $-\mathrm{C}_{6} \mathrm{H}_{6} \mathrm{O}_{3}$, devido à clivagem do anel $\mathrm{C}$ no monômero de naringerina e $\mathrm{MS}^{3} \mathrm{em} \mathrm{m} / z, 295$ [M-H-28] pela perda de $\mathrm{CO}$ (Figura 3S) (Figura $3 b) .{ }^{26} \mathrm{O}$ segundo composto desse tipo de biflavonoide foi o 7 , detentor do íon molecular em $\mathrm{m} / \mathrm{z} 637$ [M-H]', foi identificado como GB-2a-sulfato, um derivado de GB-2a com a presença do substituinte $\mathrm{SO}_{3}$, demonstrando perda de [M-H-80] e gerando íons $\mathrm{MS}^{2}$ em $\mathrm{m} / \mathrm{z}, 557$ e $\mathrm{m} / \mathrm{z}, 431$ [M-H-126-80]- característicos dos fragmentos do íon precursor GB-2a (Figura 7S). O composto 7 apresentou íon $\mathrm{MS}^{3}$ em $m / z, 295$ [M-H-136] devido a clivagem de $-\mathrm{C}_{8} \mathrm{H}_{8} \mathrm{O}_{2}$ do anel $\mathrm{B}$ e parte do anel $\mathrm{C}$ do monômero de eriodictiol, do segundo flavonoide da molécula. O composto 9 de íon molecular em $\mathrm{m} / z 719$ [M-H] foi identificado como GB-2a- $O$-glicopiranosídeo, demonstrando um íon fragmento $\mathrm{MS}^{2} \mathrm{em} \mathrm{m} / \mathrm{z} 431$ [M-H-126-162] apresentando perdas $-\mathrm{C}_{6} \mathrm{H}_{6} \mathrm{O}_{3}$ e de glicose $(\mathrm{Glc})$, assim como seu íon precursor em $\mathrm{m} / z, 557$ [M-H-162] (Figura 9S), confirmando também ser um derivado glicosilado de GB-2a., ${ }^{9,26}$

\section{Tipo GB1}

Os compostos 4 e $\mathbf{1 0}$ foram classificados como biflavonoides do tipo GB1 (flavanona-( $3 \rightarrow 8^{\prime \prime}$ )-flavonol). ${ }^{9} \mathrm{O}$ íon molecular em $\mathrm{m} / \mathrm{z}$ 573 [M-H] , correspondente ao composto 4 , foi identificado como GB-2 (naringenina- $\left(3 \rightarrow 8^{\prime \prime}\right)$-taxifolina) e pelos fragmentos $\mathrm{MS}^{2}$ com íon em $m / z, 447$ [M-H-126] novamente pela perda de $-\mathrm{C}_{6} \mathrm{H}_{6} \mathrm{O}_{3}$, devido a clivagem do anel $\mathrm{C}$ no monômero de naringerina e $\mathrm{MS}^{3} \mathrm{em}$ $m / z, 419$ [M-H-126-28] pela perda de uma molécula neutra de $\mathrm{CO}$ (Figura 3c, 4S). O composto $\mathbf{1 0}$ é um derivado glicosilado de GB-2, e denominado de GB2-7"-O-glicopiranosídeo. ${ }^{26}$ Esse composto apresenta íon molecular em $\mathrm{m} / \mathrm{z}, 735[\mathrm{M}-\mathrm{H}]^{-}$e tem como produtos de fragmentações de segunda ordem o íon em $\mathrm{m} / z$ 447 [M-H-126-162] pela perda de $-\mathrm{C}_{6} \mathrm{H}_{6} \mathrm{O}_{3}$ e glicose, assim como íon precursor em $\mathrm{m} / \mathrm{z}$ 573 característico de GB-2. O íon de terceira ordem em $\mathrm{m} / \mathrm{z} 429$ [M-H-18] apresentou perda de uma molécula neutra de água (18 Da) (Figura 10S). ${ }^{26}$

\section{Benzofenona poliisoprenilada}

A presença de benzofenona nos galhos de $P$. insignis pode ser confirmada pelo composto 5, com íon molecular em $\mathrm{m} / \mathrm{z} 601$ [M-H] , identificado como garcinielipitona FC (GFC), apresentando íon $\mathrm{MS}^{2} \mathrm{em} \mathrm{m} / z 465$ [M-H-136] pela clivagem de $-\mathrm{C}_{10} \mathrm{H}_{16}$ (136 Da) de duas unidades de isoprenos e íon $\mathrm{MS}^{3} \mathrm{em} \mathrm{m} / z, 273$ [M-H-192] pela perda de $-\mathrm{C}_{11} \mathrm{H}_{12} \mathrm{O}_{3}$ (Figura 5S). ${ }^{14,27,28}$ Garcinielipitona FC é um par tautomérico de benzofenona poliprenilada e já foi isolado do extrato hexânico das sementes de $P$. insignis, sendo suas formas isoméricas bem elucidadas. ${ }^{14,28}$

\section{Xantona}

O composto 1 foi identificado como $\alpha$-mangostin, com íon molecular em $\mathrm{m} / z, 409$ [M-H] e íons fragmentos $\mathrm{MS}^{2}$ em $\mathrm{m} / z, 351$ [M-H-58]. Para a formação desse íon de segunda ordem a molécula sofre uma desmetilação $\left(-\mathrm{CH}_{3}\right)$ formando um íon em $\mathrm{m} / z, 394$ [M-H-15].$^{29}$ Posteriormente ocorre o fechamento do anel B, com perda de $-\mathrm{C}_{3} \mathrm{H}_{7}$ formando então o íon de maior intensidade em $\mathrm{m} / \mathrm{z}, 351$ [M-H-43] . Outro fragmento de segunda ordem foi observado devido à reação competitiva durante a desmetilação, que favoreceu a perda de um grupo prenil $\left(-\mathrm{C}_{4} \mathrm{H}_{7}\right)$ no anel $\mathrm{B}$, formando também o íon de menor intensidade em $m / z, 339$ [M-H-55]. A fragmentação em MS $^{3}$ demonstrou um íon em $\mathrm{m} / \mathrm{z} 296$ [M-H-55] devido a outra perda de grupo prenil $\left(-\mathrm{C}_{4} \mathrm{H}_{7}\right)$ ligado ao anel A(Figura $\left.1 \mathrm{~S}\right)$. Esse composto foi sugerido, pois é o único isômero bem descrito por vários autores e de ampla ocorrência na família Clusiaceae e, além disso, já foi relatado em P. insignis. ${ }^{8,29}$

\section{Espectroscopia de Ressonância Magnética Nuclear de ${ }^{1} \mathrm{H}$ da subfração FAc21}

A análise por RMN de ${ }^{1} \mathrm{H}$ (Figura 12S) mostrou um sinal de dupleto com $\delta_{\mathrm{H}} 5,76$ referente ao $\mathrm{H}-2$ com constante de acoplamento, $J=12 \mathrm{~Hz}$. De acordo com a literatura, o sinal referente ao H-3 é encontrado em $\delta_{\mathrm{H}} 4,89(\mathrm{~d}, J=12 \mathrm{~Hz}$ ), porém o sinal referido foi encoberto pelo sinal da água do metanol deuterado em $\delta_{\mathrm{H}} 4,84$. A posição dos hidrogênios H-2 e H-3 evidenciam a subclasse das flavanonas. ${ }^{10,30}$

Os sinais com $\delta_{\mathrm{H}}$ em 7,11 $(d, J=8,0 \mathrm{~Hz})$ e $6,42(d, J=8,0 \mathrm{~Hz})$ foram atribuídos aos pares de átomos de hidrogênios equivalentes nas posições $2^{\prime} / 6^{\prime}$ e $3^{\prime} / 5^{\prime}$ (Figura $13 \mathrm{~S}$ ), respectivamente, confirmando o padrão de substituição característica de anel $p$-substituído da porção flavanona do biflavonoide.

Observou-se dois simpletos largos em $\delta_{\mathrm{H}} 5,97$ e 5,99 atribuídos aos hidrogênios H-6 e H-8 no anel A e um simpleto em $\delta_{\mathrm{H}} 6,25$ referente ao H-6". Outro simpleto em $\delta_{\mathrm{H}} 6,41$ é atribuído ao $\mathrm{H}-3^{\prime \prime}$ pertencente ao anel F. O H-5"' em $\delta_{\mathrm{H}} 6,92(\mathrm{~d}, J=8,0 \mathrm{~Hz})$ acopla-se com o H-6"' em $\delta_{\mathrm{H}} 7,30$ (dd, $J=8,0 \mathrm{~Hz}$ ) e esse, por sua vez, acoplase também com o $\mathrm{H}-2^{\prime \prime \prime}$ com $\delta_{\mathrm{H}} 7,35$ apresentando um simpleto largo (Figura 12S). ${ }^{10,30}$ Os sinais principais são acompanhados por sinais de menor intensidade, fato explicado pela restrição na rotação C-3 $\rightarrow$ C-8", proposta por Li et al., que evidencia o efeito atropisomérico do biflavonoide. ${ }^{10} \mathrm{~A}$ análise do espectro de $\mathrm{RMN}{ }^{1} \mathrm{H}$ e comparação com dados da literatura confirma a identificação do biflavonoide morelloflavona.

\section{Determinação do teor de fenóis totais}

Os teores de fenóis totais (FT), foram determinados em mg de equivalente de ácido gálico por grama de amostra (EAG). Os resultados das análises de FT do extrato bruto, frações Hex e AcOEt dos galhos de P. insignis estão apresentados na Tabela 3.

Tabela 3. Teor de fenóis totais ( $\mathrm{mg}$ de EAG/g de material vegetal seco) do extrato, frações Hex e AcOEt dos galhos de P. insignis

\begin{tabular}{cc}
\hline Amostras & $\begin{array}{c}\text { Fenóis Totais } \\
\left(\mathrm{mg} \mathrm{de} \mathrm{EAG} \mathrm{g}^{-1} \text { de MVS } \pm \text { DP }\right)\end{array}$ \\
\hline EEtOH & $348,3 \pm 6,5$ \\
F. Hex & $113,7 \pm 1,2$ \\
F. AcOEt & $382,0 \pm 4,7$ \\
\hline
\end{tabular}

Os resultados demonstram que o extrato bruto $(348,3 \pm 6,5 \mathrm{mg}$ de EAG $\mathrm{g}^{-1}$ ) dos galhos é rico em polifenóis, no qual pode ser confirmada pela análise de ESI-IT/MS ${ }^{n}$. A fração AcOEt $\left(382,0 \pm 4,7 \mathrm{mg}\right.$ de EAG $\left.\mathrm{g}^{-1}\right)$ apresentou uma quantidade superior ao extrato, esse alto teor corrobora com a identificação por massas de 11 compostos, dentre eles biflavonoides, benzofenona e xantona. 
Esses resultados confirmam ainda mais o alto potencial químico dos galhos de $P$. insignis.

Determinações do Fator de Proteção Solar (FPS) e comprimento de onda máximo e absorbância do extrato, frações e morelloflavona

O extrato bruto, fração AcOEt e biflavonoide morelloflavona isolado, assim como as demais frações Hex e HA, foram submetidos ao ensaio de determinação in vitro do Fator de Proteção Solar (FPS). Violante e Oliveira Junior et al. utilizaram esse método in vitro devido a sua confiabilidade e correlação com testes in vivo, uma vez que ele relaciona a absorbância da substância em questão com o efeito eritematogênico da radiação e intensidade da luz em comprimentos de onda específicos entre 290 e 320 nm (Região UVB). ${ }^{23,31}$ A Figura 4, apresenta os resultados de FPS do extrato bruto, frações Hex, AcOEt, HA, assim como morelloflavona isolada dos galhos de P. insignis.

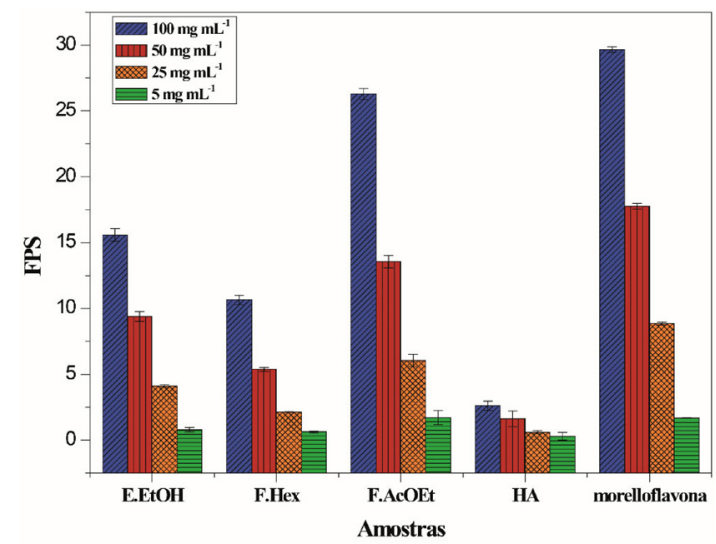

Figura 4. Fator de Proteção Solar do extrato bruto, frações Hex, AcOEt, HA e morelloflavona isolada dos galhos de P. insignis

Observa-se que o ensaio in vitro de FPS correlaciona-se diretamente com a concentração, e que o biflavonoide isolado apresentou um FPS igual a 29,66, na maior concentração, evidenciando um alto potencial fotoprotetor, assim como o E.EtOH $(15,59)$, F. Hex $(10,66)$, F. AcOEt $(26,28)$ e HA $(2,06)$. Tendo em vista que para uma espécie ser considerada com potencial fotoprotetor é necessário que ela possua os valores de FPS $\geq 2$, todas as amostras demonstraram resultados igual ou superior e são bastantes sugestivos para serem empregados em formulações cosméticas, cujo o valor mínimo aceito pela legislação brasileira, $\operatorname{RDC~}^{\circ} 30$ de 1 de julho de 2012 é de um FPS $>6 .^{31,32}$

A região de absorção da radiação ultravioleta (UV), também é um fator bastante relevante para as substâncias a serem empregadas como filtros solares, uma vez que eles irão proteger a pele dos raios UVA (320-400 nm), UVB (290-320 nm) ou UVC (200-290 nm). ${ }^{23} \mathrm{~A}$ Tabela 4 apresenta o comprimento de onda máximo $\left(\lambda_{\max }\right)$ e a região de absorção de cada amostra em diferentes concentrações, assim como a Figura 5 apresenta os perfis de absorção espectrofotométrica para o extrato, frações e morelloflavona isolada.

Tabela 4. Comprimento de onda de absorção máxima $\left(\lambda_{\max }\right)$ e tipo de absorção UV do extrato, frações e morelloflavona isolada dos galhos de $P$. insignis

\begin{tabular}{lcc}
\hline Amostras & $\lambda_{\text {max }}(\mathrm{nm})$ & Tipo de absorção \\
\hline E.EtOH & 289 & UVB \\
F.Hex & 276 & UVC \\
F.AcOEt & 290 & UVB \\
F.HA & 289 & UVB \\
Morelloflavona & $290-355$ & UVA-UVB \\
\hline
\end{tabular}

UVA (320-400 nm), UVB (290-320 nm) e UVC (200-290 nm).

\section{Atividade antioxidante frente ao radical DPPH}

Um antioxidante ou bioantioxidante pode ser caracterizado como uma substância que, presente em baixas concentrações, quando comparada com um substrato oxidável, diminui significativamente a oxidação do referido substrato. ${ }^{33,34}$ Grande parte desses compostos antioxidantes pode ser encontrada nas frutas, legumes e plantas
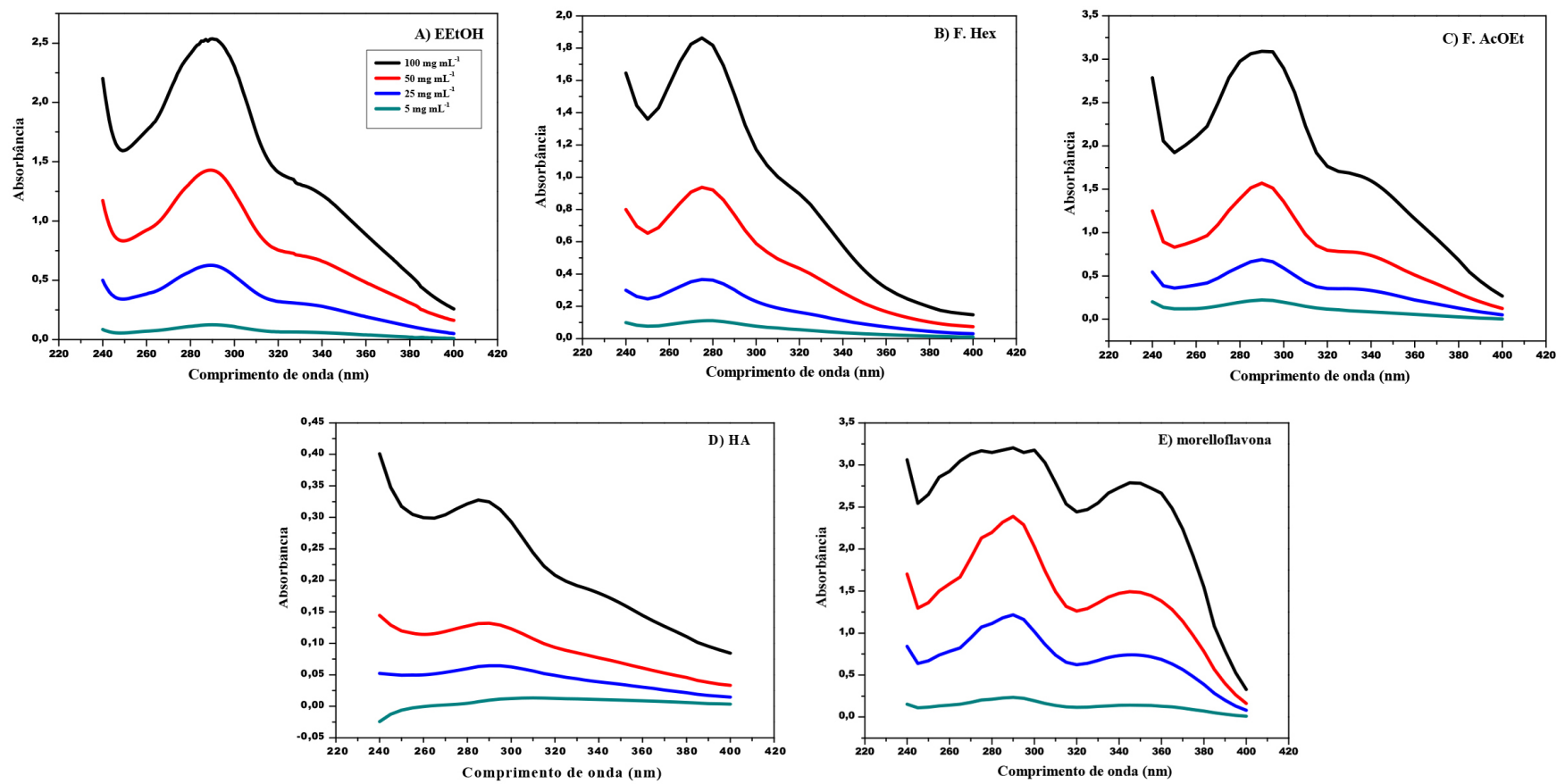

Figura 5. Perfil de absorção espectrofotométrica do extrato (A), frações Hex (B), AcOEt (C), HA (D) e morelloflavona (E) isolada dos galhos de P. insignis (240-400 nm) 
com a função de prevenir doenças neurodegenerativas como câncer, cardiovasculares e envelhecimento precoce. ${ }^{33,35}$

O E.EtOH, frações Hex e AcOEt e morelloflavona dos galhos de $P$. insignis apresentaram resultados promissores frente ao radical DPPH, obtendo-se o percentual de atividade antiradicalar (\%AA) e a concentração eficiente em mg mL $\mathrm{m}^{-1}\left(\mathrm{CE}_{50}\right)$ (Tabela 5) (Figura 6). Observou-se que o extrato EtOH, F.AcOEt e morelloflavona isolada apresentaram um \%AA superior a $60 \%$, atingindo máximos de $90,57,90,88$ e $89,93 \%$, respectivamente, na maior concentração (250 $\left.\mu \mathrm{g} \mathrm{mL}^{-1}\right)$. Quando comparados ao padrão BHT $(89,88 \%)$, ambos foram equivalentes. A concentração eficiente média para as amostras encontram-se na Tabela 5, no qual se observou que E.EtOH e a morelloflavona apresentaram $\mathrm{CE}_{50}$ de 59,87 e $52,32 \mu \mathrm{g} \mathrm{mL} \mathrm{L}^{-1}$, respectivamente, sendo mais eficientes quando comparado com o padrão BHT $\left(69,34 \mu \mathrm{g} \mathrm{mL}^{-1}\right)$, na redução de $50 \%$ da concentração do radical.

Tabela 5. Concentração eficiente média $\left(\mathrm{CE}_{50}\right)$ e percentual de atividade antiradicalar (\%AA) do extrato etanólico, frações e morelloflavona isolada dos galhos de $P$. insignis

\begin{tabular}{lcc}
\hline Amostras & $\mathrm{CE}_{50}\left(\mu \mathrm{g} \mathrm{mL}^{-1}\right)$ & $\% \mathrm{AA} \pm \mathrm{DP}\left(250 \mu \mathrm{g} \mathrm{mL}^{-1}\right)$ \\
\hline E. EtOH & $59,87 \pm 0,80$ & $90,57 \pm 0,81$ \\
F. Hex & $120,16 \pm 11,92$ & $78,83 \pm 7,51$ \\
F. AcOEt & $99,24 \pm 5,58$ & $90,88 \pm 0,95$ \\
Morelloflavona & $52,32 \pm 0,62$ & $89,83 \pm 0,25$ \\
Rutina & $47,06 \pm 4,65$ & $94,14 \pm 0,22$ \\
BHT & $69,34 \pm 5,53$ & $89,88 \pm 0,83$ \\
\hline
\end{tabular}

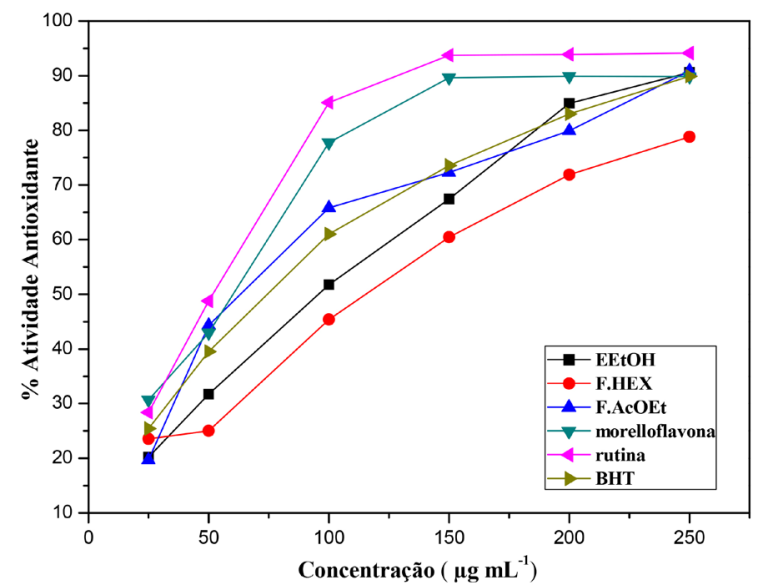

Figura 6. Porcentagem de AA do extrato etanólico e das frações Hex, AcOEt e morelloflavona isolada dos galhos de P. insignis e dos padrões rutina e BHT

\section{CONCLUSÕES}

O estudo realizado permitiu caracterizar pela primeira vez o perfil químico dos galhos de $P$. insignis, contribuindo para o estudo metabolômico da espécie. A análise realizada por ESI-IT/MS ${ }^{n}$ indica que o gênero Platonia é altamente rico em polifenóis, com destaque para elevada quantidade de biflavonoides, que evidenciam um alto potencial fotoprotetor e antiradicalar. Os resultados dos ensaios in vitro trazem um importante ponto de partida para o desenvolvimento de produtos funcionais para o setor farmacêutico e alimentício.

\section{MATERIAL SUPLEMENTAR}

Os dados espectrométricos do extrato, fração AcOEt e subfração FAc21 estão disponíveis em http://quimicanova.sbq.org.br, em formato pdf, com acesso livre.

\section{AGRADECIMENTOS}

Os autores agradecem ao CNPq, INCTBioNat (2018/406126-6, 2014/ 465637-0) pelo apoio financeiro. Agradecem também à FAPEPI (EDITAL FAPEPI/ MCT/ CNPq/ CT-INFRA No 007/2018 - Programa PPP) pelo auxílio financeiro concedido a S. D. L. Freitas (Termo de concessão $n^{\circ}$ 043/2019).

\section{REFERÊNCIAS}

1. Forzza, R. C.; Baumgratz, J. F.; Bicudo, C. E. M.; Canhos, D. A. L. C,; Anibal, A.C.; Marcus, A. N.; Costa, A. F. C.; Hopkins, D. P.; Leitman, M. G.; Logmann, L. G.; Lughadha, E. N.; Maia, L. C. M.; Menezes, G.; Peixoto, M. P.; Pirani, A. L.; Prado, J. R.; Queiroz, J. Souza, L. P.; Souza, S.; Castro, V.; Stehmann, J. R.; Sylvestre, L.; Walter, S.; Bruno, M. T.; Zappi, D. C.; Bioscience 2012, 62, 39.

2. Zeni, A. L. B.; Parisotto, A. V.; Mattos, G.; Helena, E. T. S.; Ciência \& Saúde Coletiva 2017, 22, 2703.

3. Bruning, M. C. R.; Mosegui, G. B. G.; Vianna, C. M. M.; Ciência \& Saúde Coletiva 2012, 17, 2675.

4. Stevens, P. F. Em The Families and Genera of Vascular Plants. vol. IX. Clusiaceae-Guttiferae; Kubitzki, K., eds; Springer-Verlag: Berlin Heidelberg, 2007, cap. 8.

5. Yamaguchi, K. K. L.; Pereira, C. V. L.; Lima, E. S.; Veiga Júnior, V. F.; Scientia Amazonia 2014, 3, 39.

6. Forzza, R. C; Baumgratz, J. F. A.; Bicudo, C. E. M.; Carvalho Júnior, A. A.; Costa, A.; Costa. D. P.; Hopkins, M., Leitman, P. M.; Lohmann, L. G.; Maia, L. C.; Martinelli, G.; Menezes, M.; Morim, M. P.; Coelho, M. A. N.; Peixoto, A. L.; Pirani, J. R.; Prado, J.; Queiroz, L. P.; Souza, V. C.; Stehmann, J. R.; Sylvestre, L. S.; Walter, B. M. T.; Zappi, D.; Catálogo de plantas e fungos do Brasil, Instituto de Pesquisas Jardim Botânico do Rio de Janeiro: Rio de Janeiro, 2010, v. 2.

7. Gasparato Júnior, A.; Ferreira, I. C. P.; Nakamura, C. V.; D Filho, B. P.; Jacomassi, E.; Young, M. C. M.; Cortez, D. A. G.; Acta Farm. Bonaerense 2005, 24, 371.

8. Costa Júnior, J. S.; Ferraz, A. B. F.; Sousa, T. O.; Silva, R. A. C.; Lima, S. G.; Feitosa, C. M.; Cito, A. M. G. L.; Cavalcante, A. A. C. M.; Freitas, R.; Sperotto, A. R. M.; Peres, V. F.; Moura, D. J.; Saffi, J.; Basic Clin. Pharmacol. Toxicol 2013, 112, 34.

9. Ferreira, R. O.; Carvalho, M. G.; Silva, T. M. S.; Quim. Nova 2012, 35, 2271.

10. Li, X. C.; Joshi, A. S.; Tan, B.; Elsohly, H. N.; Walker, L. A.; Zjawiony, J. K.; Ferreira, D.; Tetrahedron 2002, 58, 8709.

11. Souza Cunha, A.; Alves, Moraes, M. M.; Brito, L. M.; Oliveira, L. G. C.; Sobrinho-Júnior, E. P. C.; Costa, I. C. G.; Freitas, S. D. L.; Rodrigues, K. A. F.; Chaves, M. H.; Arcanjo, D. D. R.; Carvalho, F. A. A.; J. Evidence-Based Complementary Altern. Med. (2017), doi: $10.1155 / 2017 / 3126458$.

12. Lima, M. C.; Bacuri: agrobiodiversidade, $1^{\text {a }}$ ed., Instituto Interamericano de Cooperação para a Agricultura: São Luís, 2007. Nascimento, W. M. O.; Carvalho, J. E. U.; Müller, C. H.; Rev. Bras. Frutic. 2007, 29, 657.

13. Santos Júnior, R. Q.; Soares, L. C.; Maia Filho.; A. L. M.; Araujo; K. S.; Santos, I. M. S. P.; Costa Júnior, J. S.; Saffi, J.; ConScientiae Saúde 2010, 9, 575.

14. Costa Júnior, J. S.; Ferraz, A. B. F.; Filho, B. A. B.; Feitosa, C. M.; Cito, A. M. G. L.; Freitas, R. M.; Saffi, J.; J. Med. Plant. Res. 2011a, 5 , 293; Costa, I. C. G.; Dissertação de Mestrado, Universidade Federal do Piauí, Brasil, 2014; Nascimento, J. L.; Coelho, A. G.; Barros, Y. S. O.; Silva, O. A.; Freitas, R. M.; Rocha, M. S.; David, J. M.; Costa Júnior, J. S.; Rufino, D. D. A.; Arcanjo, D. D. R.; Costa Junior, J. S.; Moura, L. H. P.; Ferraz, A. B. F.; Rossatto, R. R.; Quintans Júnior, L. J.; Oliveira, R. C. M.; Citó, A. M. D. G. L.; Oliveira, A. P.; Nat. Prod. Res. 2014, 28 , 
923; Pinheiro, E. E. A.; Oliveira, F. A. A.; Lima Neto, J. S.; Mesquita, A. B. S.; Brito, C. A. R. S.; Figueirêdo, G. S.; Ferreira, J. H. L.; Barreto, H. M.; Citó, A. M. G. L.; Revista Brasileira de Biodiversidade e Biotecnologia 2015, 1, 1; Rocha, E. S.; Dissertação de Mestrado, Universidade Federal do Tocantins, Brasil, 2017.

15. Costa Júnior, J. S.; Almeida, A. A. C.; Tome, A. R.; Citó, A. M. G. L.; Saffi, J.; Freitas, R. M.; Epilepsy \& Behavior 2011, 22, 678.

16. Sousa, C. M. M.; Dissertação de Mestrado, Universidade Federal do Piauí, Brasil, 2012.

17. Lustosa, A. K. M. F.; Bezerra, E. A.; Rodrigues, K. A. F.; Amorim, L. V.; Lima Neto, J. S.; Araújo, B. Q.; Costa Junior, J. S.; Mendes, A. N.; Carvalho, F. A. A.; Arcanjo, D. D. R.; Citó, A. M. D. G. L.; Rev. Cubana Plant. Med. 2018, 23, 2018.

18. Rufino, M. S. M.; Alves, R. E.; Brito, E. S.; Pérez-Jiménez, J.; Sauracalixto, F.D.; Mancini-Filho, J.; Food Chem. 2010, 121, 996.

19. Viana, A. F. S. C.; Reis Filho, A. C.; Lima, G. S.; Diniz, J. A.; Santos, M. O.; Oliveira, R. C. M.; Anais do I Encontro Estratégico em Ciências Farmacêuticas 2013, 4, 129.

20. Uriu, D. M.; Godoy, B. S. A.; Battirola, L. D.; Andrighetti, C. R.; Marques, M. I.; Valladao, D. M. S.; Rev. Árvore (2018), doi. org/10.1590/1806-90882017000300016.

21. Origin (Pro), Versão 8.0; OriginLab Corporation; Universidade Federal do Piauí, Brasil, 2019.

22. Mansur, J. S.; Breder, M. N. R.; Mansur, M. C. A.; Azulay, R. D.; An. Bras. Dermatol. 1986, 61, 121.

23. Oliveira Júnior, R. G.; Araújo, C. S.; Souza, G. R.; Guimarães, A. L.; Oliveira, A. P.; Lima-Saraiva, S. R. G.; Morais, A. C. S.; dos Santos, J. S. R.; Almeida, J. R. G. S.; J. Appl. Pharm. Sci. 2013, 3, 122.

24. Sayre, R. M.; Agin, P. P.; Levee, G. J.; Marlowe, E.; Photochem Photobiol. 1979, 115, 46.
25. Boeira, C. P.; Piovesan, N.; Soquetta, M. B.; Flores, D. C. B.; Lucas, B. N.; Rosa, C. S.; Terra, N. N.; Ciênc. Rural 2017, 11, 48.

26. Carrillo-Hormaza, L.; Ramírez, A. M.; Quintero-Ortiz, C.; Cossio, M; Medina, S.; Ferreres, F.; Gil-Izquierdo, A.; Osorio, E.; J. Funct. Foods 2016, 27, 503; Akpanika, G. A.; Winters, A.; Wilson, T.; Ayoola, G. A.; Adepoju-Bello, A. A.; Hauck, B.; Food Chem. 2017, 222, 35.

27. Costa Júnior, J. S.; Feitosa, C. M.; Citó, A. M. G. L.; Freitas, R. M.; Henriques, J. A. P.; Saffi, J.; J. Biol. Sci. 2010, 10, 747.

28. Silva, A. P. S. C. L.; Oliveira, G. L. S.; Medeiros, S. C.; Sousa, A. M. L.; Lopes, L. S.; David, J. M.; Junior, J. S. C.; de Freitas, R. M.; Phytomedicine 2016, 23, 477; Wu, C.; Lu, Y.; Wei, B.; Yang, S.; Won, S.; J. Nat. Prod. 2008, 71, 246.

29. Azebaze, A. G. B.; Dogmo, A. B.; Meyer, M.; Ouahouo, B. M. W.; Valentin, A.; Laurenguemfo, E.; Nkekgfack, A. E.; Vierling, W.; Ann. Trop. Med. Parasitol. 2007, 101, 23; Mahabusarakam, W.; Wiriyachitra, P.; J. Nat. Prod. 1987, 50, 475; Wittenauer, J.; Falk, S.; SchweiggertWeisz, U.; Carle, R.; Food Chem. 2012, 134, 445.

30. Santos, C. C.; Oliveira, R. R.; Figueiredo, M. R.; Rev. Fitos 2007, 3, 32. 31. Violante, I. M. P.; Souza, I. M.; Venturini, C. L.; Ramalho, A. F. S.; Santos, R. A. N.; Ferrari, M.; Rev. Bras. Farmacogn. 2009, 19, 452.

32. http://bvsms.saude.gov.br/bvs/saudelegis/anvisa/2012/ rdc0030_01_06_2012, acessada em abril 2021.

33. Oliveira, G. L. S.; Silva, A. P. S. C. L.; Feitosa, C. M.; Almeida, F. R. C. Em Antioxidantes: aspectos químicos, farmacológicos e terapêuticos; Feitosa, C. M., eds.; Editora Átomo: Campinas, 2017, cap.1.

34. Oroian, M.; Escriche, I.; Food Res. Int. 2015, 74, 10.

35. Stangenberg, S.; Int. J. Biochem. Cell Biol. 2015, 64, 81. 